\title{
Mass Customization: a critical perspective on parametric design, digital fabrication and design democratization
}

\section{SIGRADI2018 TECHNOPOLITICAS \\ xxii congresso da sociedade iberoamericana de gráfica digital 22th conference of the iberoamerican society of digital graphics 07|08|09|novembro|2018 iau usp | são carlos | sp br}

\author{
Mateus van Stralen \\ UFMG | Brazil | mateus-stralen@ufmg.br
}

\begin{abstract}
The ability of parametric design to generate variations and bespoke products, combined with the capability of digital fabrication to render this variety physical enables the mass-production of non-standard products. Several companies are adopting parametric driven digital interfaces that enable the user to change design parameters to personalize a product. This "democratization" of design - as it is being called - has multiple social, cultural, and design implications. This paper addresses the idea "design democratization" with a critical viewpoint and advocates for a different perspective of design democratization based on conversation cycles and the copying, transforming, and sharing of code.
\end{abstract}

Keywords: Mass Customization, personalization, design democratization

\section{INTRODUCTION}

In what is for some a model situation architects that deals with single clients can understand his tastes, culture, beliefs, and needs. He can make decisions for the client, orientate his thoughts, or give him technical support. Ideally, he can also engage in conversation with his client to generate new thoughts and ideas that will result in a product not foreseen by both. In this case the designed object, when the fruit of meaningful conversations, will be a unique reflection on both architect and client. However, the majority of what is being built today with the participation of the architect is not exposed to the full creative potential of the human environment. High rise buildings with standard apartments and even whole neighborhoods of standard houses are typical in almost every country. Without a specific client the architect designs for a standard user, classifying him according to his social profile. Large-scale solutions are still based on the functionalist paradigm of standardization, where the house is seen as a machine that can be replicated ad infinitum in a production line, built for a standard user with effectively no variety. This system has been broadly criticized in the last decades but is still the primary strategy of production of the architectural space. Nevertheless, the contemporary confluence of parametric design with digital fabrication offers designers the possibility to create new design and production processes.

The ability of parametric design to generate variations and bespoke products where the users can manipulate the parameters, combined with the capability of digital fabrication to render this variety physical is perhaps one of the most profound aspects of the contemporary use of computation in architecture. Many authors see the promise to deliver variety and personalization with efficiency and economy of scale as a response to the
Fordian paradigm of mass production and standardization. Within this context, discussions concerning parametric design and digital fabrication are frequently associated with what Stan Davis (1987) has called "Mass Customization." The concept, latter framed and defined by Joseph Pine (1993), refers to "the mass production of individually customized goods and services" combining the idea of personalization with the low cost associated with standardization.

The concept of mass customization has different implications in different sectors ranging from the customization of goods, services, and experiences. It may operate in different production levels - design, production or post-production. In architecture, the idea of combining economy of scale with personalization is not new. Most buildings combine standard mass-produced components to generate non-standard personalized designs. Even mass-produced houses and apartments can be customized at the production level, as many companies offer the possibility for their clients to change the layout, colors, and other features of the interiors. Nevertheless, exploring mass customization at the design level still represents a challenge that is being met by the creation of parametric designs with dynamic parameters that can be altered by the user. The parametric definitions of products' geometry can be made accessible via digital interfaces to anyone to design their versions of the product. This "democratization" of design - as it is being called by many (Kolarevic, 2015) - is receiving increased attention and has multiple social, cultural, and design implications. Nevertheless, many design interfaces associated with the idea of design democratization and personalization does no appear to represent the one or the other, leading to a false idea of democratization. Therefore, this paper addresses possible skepticism towards the idea of "design democratization" with the purpose of avoiding a false 
understanding of mass-customization and personalization in design.

The methodology proposed for this article is a literature review associated with the description and analyses of different examples of mass-customization interfaces. Those examples range from the design of small objects, such as proposed by Gramazio and Kohler, Assa Ashuach, Nervous Systems, the design of meta-designs, such as in Sketchchair, Mattermachine, and to the design of houses, such as in Chassis House and Wikihouse.

\section{DIGITAL FABRICATION, PARAMETRIC DESIGN AND CUSTOMIZATION}

Parametric is a general term used in a variety of disciplines and means something that "relates to or is expressed in terms of a parameter or parameters" ("parametric | Definition of parametric in English by Oxford Dictionaries," n.d.). In design, there is no precise definition of the term, and its use has varied according to time and context (Davis, 2013). Several authors claim that all design can be seen as parametric (Aish and Woodbury, 2005, Gerber, 2007) because all designs use parameters whereas others see it as a style (Schumacher, 2008). In general, the term is frequently associated with the generation of complex forms and to the utilization of parametric models. In traditional design processes those that use CAD programs as an extension of the drawing board and explicit modeling techniques - the change of parameters, like the geometry of a room, could only be implemented by suppression and reconstruction. This process is rethought in parametric modeling, where the model can be defined as a set of geometric associations that are applied through parametric expressions and constraints. This chain of geometrical relations can be manipulated without losing the consistency of the whole. As an effect, it increases the designer's ability to explore variations and change by diminishing the time spent in reworking the model to generate multiple bespoke outcomes. If these parameters can be manipulated dynamically - with the creation of dynamic parameters, this opens the possibility to involve the user in the design process.

In a parametric model, inputs are interwoven to specific outputs through an encodement model of communication (communication theory). If some information is changed in one part of the model this affects the whole model. Because the encodement model of communication does not involve meaning, many authors regard parametric models as neutral tools. However, as Woodbury (2010) points out, "the way in which data flows deeply affects the designs possible, and how a designer interacts with them." The choice of inputs and outputs and how they relate to one another is a design decision. In the same direction, Benjamin (2012) advocates that the "process of evaluating a parametric model reinforces the need for clearly defining design objectives (also called fitness criteria). Design objectives are values: they are the goals and desires of a project that involve judgment and beliefs, outside of efficiency and computation."

In its turn, digital fabrication can be defined as "a way of making that uses digital data to control a fabrication process" (Iwamoto, 2009). The association of CAD and CAM technologies is narrowing the gap between traditional forms of representation and building. It relies on Computer Numerical Control (CNC) tools to build, cut or print parts. These tools remount to the first Numerically Controlled (NC) machines, such as portrayed by Hartley E. Howe (1955) in his article: Teaching power tools to run themselves" (Figure 1). The article starts with the story of Joe, who wanted to make a new set of dining-room chairs. Instead of making one manually, he rents a batch of punch-card tapes containing the codes for the design of the chair and runs them on an NC machine. According to Howe (1955), the main advantage of the Numerically Controlled Machine was the ability to rapidly make different objects and parts without changing its general configuration - in other words, it could be used to make custom objects. Furthermore, another advantage was that the design codes could be recorded magnetically so that a machine owner did not have to own a computer - he could buy, rent or share each design.

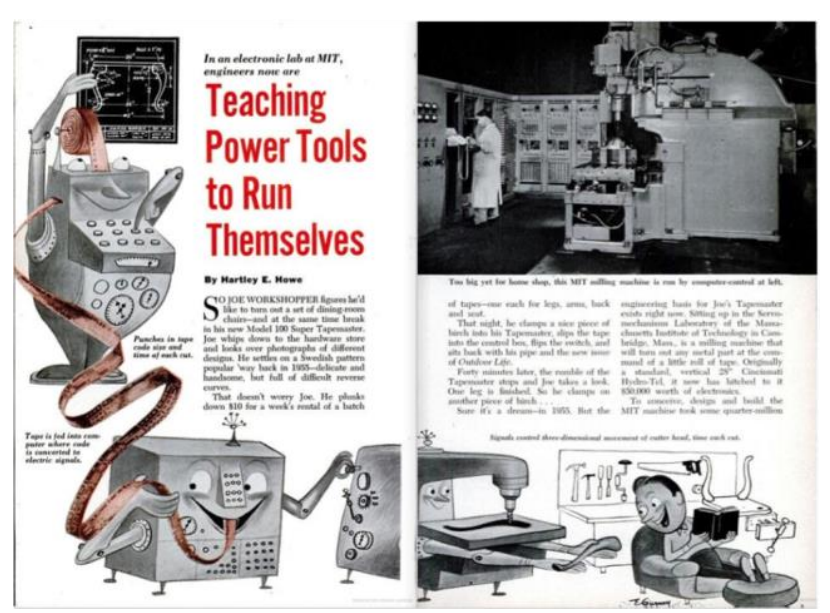

Figure 1: Two pages of Hartley E. Howe (1955) article published in Popular Science about the Numerically Controlled Milling Machine named Mass. (Howe, 1955).

During the first years of development, those machines were not envisioned to be used by or for designers, and most explorations were directed towards aerospace engineering. It would take a few decades before it could be integrated into a building workflow. Although there were significant technological developments during the following decades, such as the evolution and integration of computers in the process, the main concepts, potentials, and expectations did not change much since the 1950s. The now called Computer Numerically Controlled (CNC) machines have passed from room-size scale to desktop proportions. It adopts digital coding instead of radio transmissions and can make use of the internet to send or download digital files from all over the world. Designs are shared on various websites, and many can be freely downloaded. The idea of customization is taken a step further where people can download a design, make adaptations and "print" it at home. Machine workshops, frequently called maker-spaces, have many different CNC machines at their disposal and can produce complex objects. CNC tools enable to translate data almost directly to physical objects, materials, and structures in such a way and speed that would have been impossible without computational capabilities. In a CNC machine, making several copies of an object is almost the same effort as making several different objects. 
Therefore, they are proper tools to custom-produce objects. It not only provides a medium for creating different iterative cycles with rapid prototyping techniques, but also enables the creation of innovative design methods, assembly, and fabrication processes. In some sense, Howe's predictions were fulfilled and even surpassed.

\section{MASS CUSTOMIZATION, PRODUCT CONFIGURATORS AND DESIGN DEMOCRATIZATION}

From a market perspective, consumers have become more demanding for variety, uniqueness, and personalization. The strategy "a few sizes fit all," from mass manufactures, leaves many users dissatisfied with products they buy because users needs are heterogeneous (Von Hippel, 2005). To address these issues companies began to focus on understanding and fulfilling the needs of individual customers. In architecture, several companies offer the possibility of configuring houses using online digital interfaces. Those systems are generally associated with specific design strategy that combines standard mass-produced components to generate a non-standard personalized design. For Noguchi et al. (2016, p.112), the design interface is part of a service (S) that combines designing, producing and marketing a product. The housing components are labeled as products $(\mathrm{P})$ covering production techniques such as modularization. Mass customization (MC) can thus be modeled as $M C=f(S P)$ (Noguchi et al., 2016). In this system, the degree of control offered to the user frequently depends on the design constraints determined in $\mathrm{P}$.

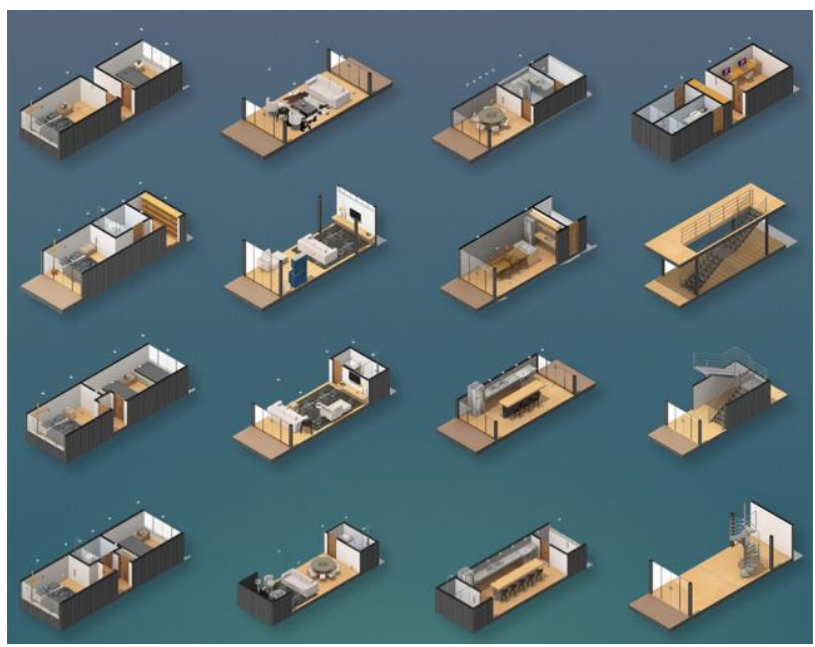

Figure 2. 16 modules of Chassis house (Hometeka, 2014).

An example of a customizable house system developed within this logic is Hometeka's Chassis House (figure 2), where the architects developed 16 initial modules that can be recombined to generate spatial variations and different plans. The modules can be prefabricated and transported to the site. Other examples that use modular processes for mass customization are Resolution: 4 Architecture's (RES4) Prefab Homes, and BluHomes with its 3D configurator interface. For Kolarevic (2015), this kind of process offer ways to customize predefined house designs, but do not offer dimensional customization with the possibility to manipulate the house's overall geometry online. To enable "true" customization, he proposes that a mass customizable house should be "parametrically defined, interactively designed (via a website or an app), and digitally prefabricated, using file-to-factory processes." (Kolarevic, 2015, p.52). In this way, custom houses would be available for a broader segment of society, where users could make design decisions based on open-parameters defined by the architect. This handling of decisions to the users would lead, according to Kolarevic (2015), to a "democratization" of the design process.
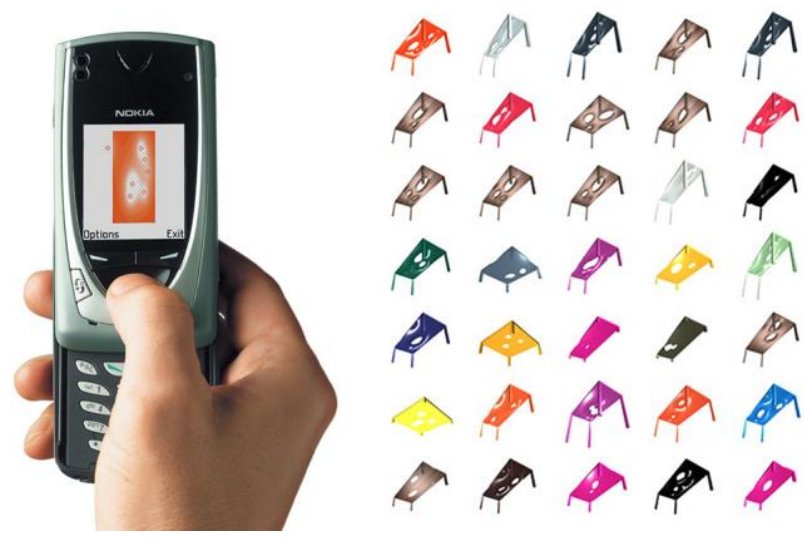

Figure 3: Gramazio and Kohler's mTable. (Gramazio and Kohler, 2012).

Nowadays, there are many examples of design interfaces that associate parametric design and digital fabrication to enable people to mass customize designs and products. These interfaces can be differentiated according to the degree of control they offer to the user. At least four groups can be distinguished. The first group is characterized by design interfaces that offer a certain number of open parameters for the user to customize a given product. Those interfaces are frequently called "configurators," as they offer the possibility of configuring a predefined design by changing some attributes. Examples of this first group are Gramazio and Kohler's mTable (2002), Assa Ashuach Co-design objects, Nervous Systems design systems, and Hermit Houses. mTable (2002) is a customizable design system where the user can make holes by placing deformation points in the tabletop of a parametrically variable table design (size, dimension, material, and color). (Figure 3)

In its turn, Assa Ashuach's Co-design (figure 4) is based on his concept of Digital Forming, a design system that involves software for customizing products and additive manufacturing method $(\mathrm{P})$. This system allows product personalization and reconfiguration within an online interface, connecting the user, designer, and manufacturer. Each object has different open parameters, but in general most designs available online involve the change of color, pattern, and size. Likewise, Nervous System offers several online design apps (figure 5) where users can change the design parameters and customize different products such as jewelry and puzzles. Some design interfaces created by the group offer the option for the user to download the product and print it at home using additive manufacturing (3D printers). Hermit Houses, from The Cloud Collective, is an example of the possibility of designing mass-custom houses using dimensional variation. The project associates an online 

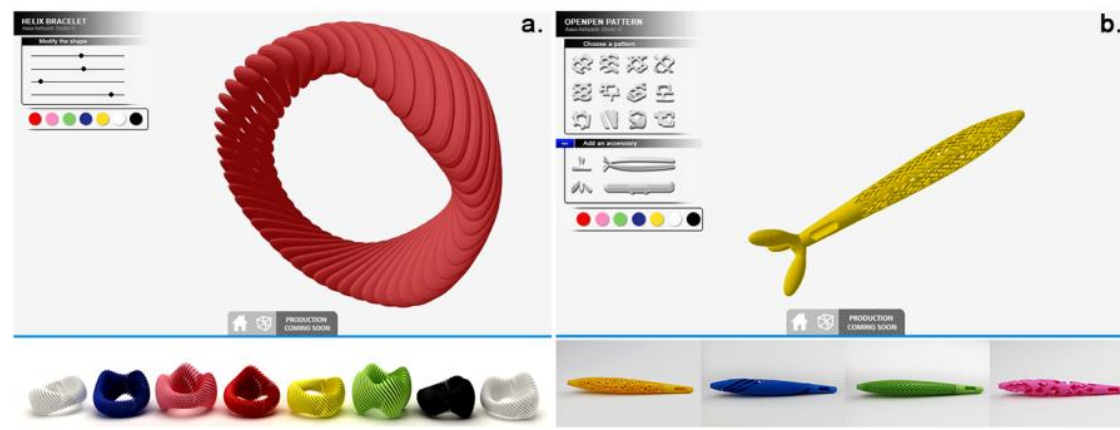

b.

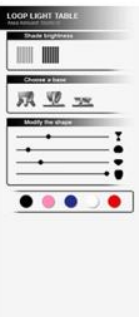

Figure 4: Assa Ashuach (2017) design interfaces: Heliz Bracelet, Openpen Patter, and Loop Light Table. (Ashuach, 2017).

interface (S) where the user can configure different dimensional dynamic-parameters (figure 6) with a digital fabrication process $(P)$. The interface generates 3D drawings for visual feedback, construction documentation, and files for the CNC fabrication process.

The second and more promising group of custom design systems is related to design interfaces that enable technical feedback between system and designer. Those interfaces can be seen as a simplified version of "design amplifiers" put forward by Negroponte (1975, p.108) where the system contributes with technical expertise to the designer's intentions. Those design systems do not only offer a choice of dynamic-parameters to the user but more importantly, empowers him to act.

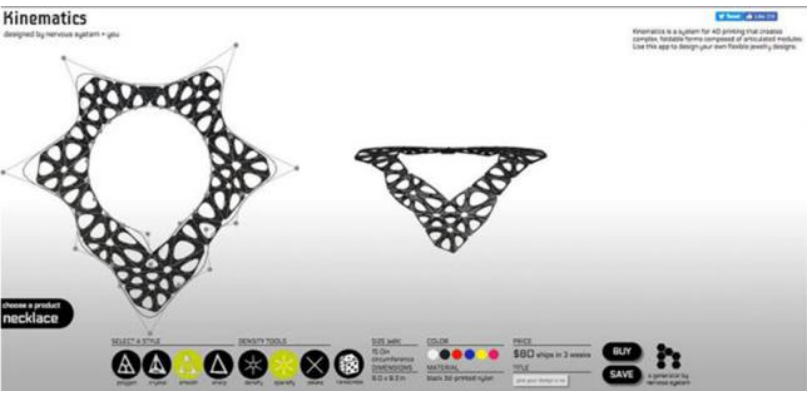

Figure 5: Nervous System design interfaces. (Nervous system, 2017). (Nervous Systems, n.d.).

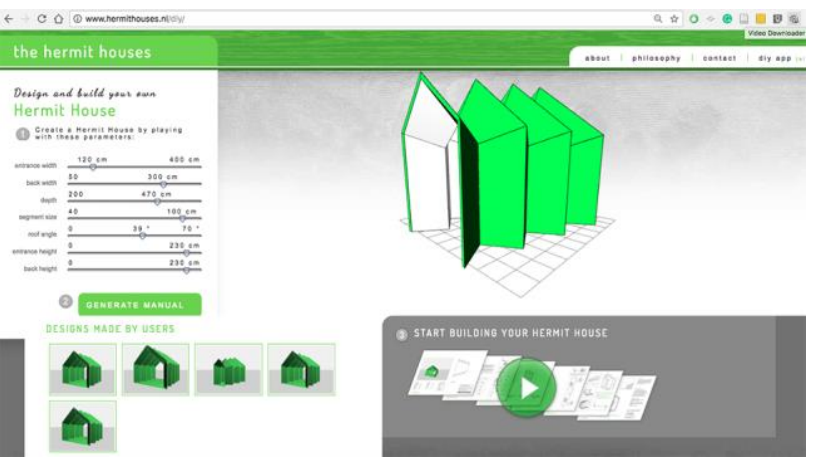

Figure 6: Hermit houses online configurator. (The Hermit houses, n.d.).

Sketchchair can be seen as an example of such interface. It is a design interface (figure 7) constructed to facilitate the design of chairs intended for digital fabrication. The open source system enables the user to control, in a straightforward manner, the whole process, from design, evaluation, detailing and manufacturing. The design principle is based on $2 \mathrm{D}$ sections, where the object is sectioned in the longitudinal and transversal directions forming interlocking planes. The user can easily draw the profile of the chair and later adjust the layers and sections to determine the final shape. The software also includes a feedback system that enables to test the ergonomy and to simulate gravitational forces. The product is a vector file with all the pieces of the chair that can be sent to a CNC cutting machine or even printed as a mold and cut by hand. The system was tested in a design workshop that revealed that people express a preference for their own design rather than those sold in stores and indicated that they would consider their chair less disposable (Saul et al. 2012).

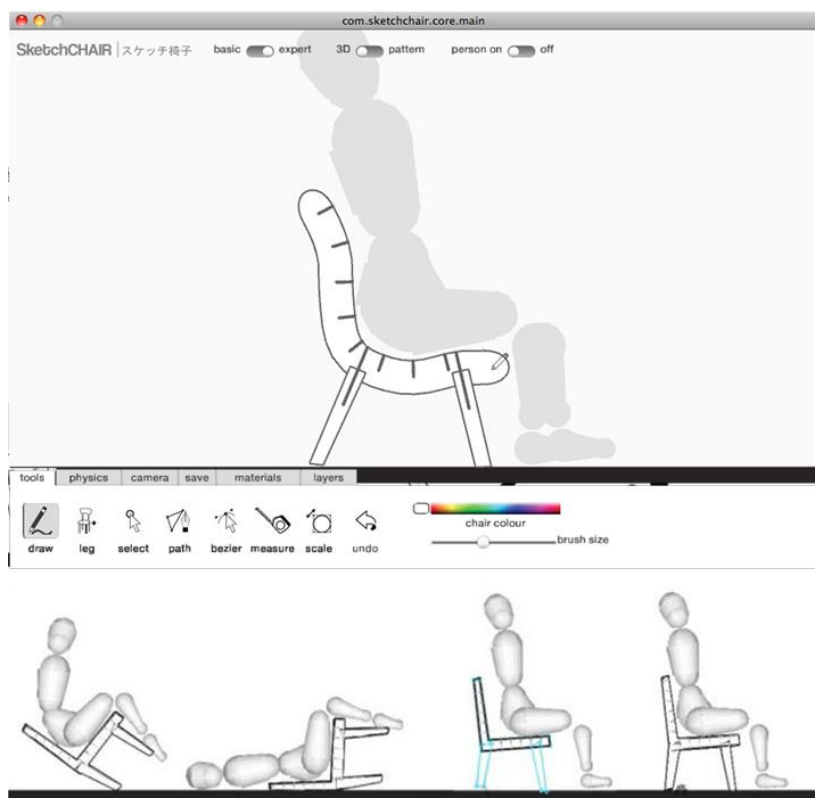

Figure 7: Sketchchair Interface, and gravity simulation (Saul et al. 2012).

The third group is characterized by design interfaces developed to enable the creation of design interfaces meta-designs. Mattermachine is an example of such interface that offers a parametric design platform to allow designers to create and provide customizable products over the internet. The design interface (figure 8) consists of a node-based editor where one component can be connected to another to form a parametric definition. The designer can create open-parameters for the user to customize the object according to his need. In this way, the design can be constructed dynamically, where the user defines the final parameters of the object. In this 
process, the degree of openness of the object to the intervention of the user is stipulated by those who establish the initial parameters. However, the user can also opt to make more profound changes in the design by assessing the parametric chain of constraints. The online design platform enables both forms of interaction with the use of two different interfaces, one with the open parameters, called presentation mode, and the other with the parametric definition of the object. Mattermachine stores a database with all designed objects which the user can access, change the parameters, and make the product available again generating a collaborative process and enabling dialogue between different designers. A designer can choose to distribute his designs for free or charge an access fee to the cut layout or codes for 3D printing. The program also allows different people to work simultaneously on the creation of the same model, allowing a greater collaboration between designers, engineers, manufacturers, among others.

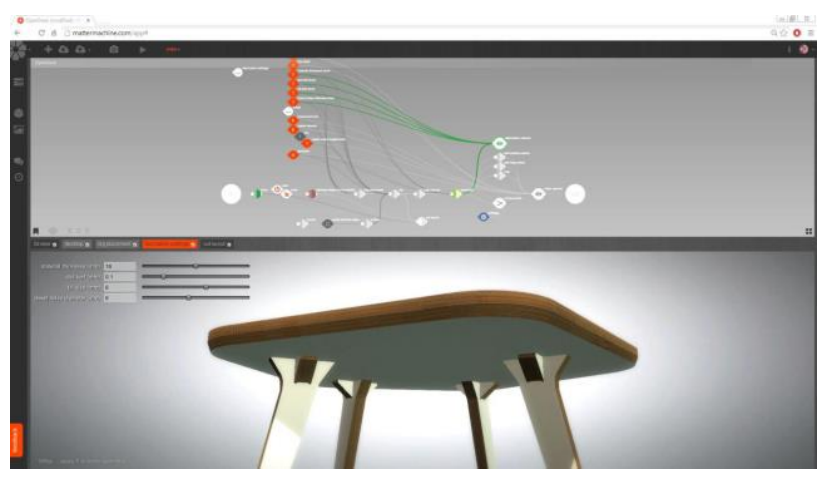

Figure 8: Mattermachine Interface with the node-based design programming environment above and the open parameters below. (Mattermachine, 2017).

\section{"MENU-PICKING" AND THE "INNOVATION USER"}

Most design interfaces presented can be associated with what is called a database design where the designer creates a drawing space for inexperienced users. The creation of these meta-designs may represent a possible alternative to the increasing control exercised by architects of the whole process of design and construction, made possible by the association between digital design and digital manufacturing technologies. However, if the number of open-parameters is not enough to create complex interactions and meaningful changes, the design process will be likely condemned to be what Negroponte (1975, p.115) calls a "menu-picking activity." For Negroponte (1975) having a list of options - a "menu of solutions" - does not correspond to a design activity. In this case, the menu of solutions is not much different from going to a pizza restaurant and select different toppings for a pizza within a restricted set of options. If advanced computer-controlled fabrication methods make these pizzas the consumer may receive the pizza faster and with a precise shape, but this does not, in principle, improve what he receives. If he makes a bad combination of flavors, he has to cope with the consequences of his choice. Looking from this perspective, objects can fit better, but this does not guarantee emotional attachment (Norman, 2004). As Norman (2004, p.220) puts it, "things do not become personal because we have selected some alternatives from a catalog of choices. To make something personal means expressing some sense of ownership, of pride. It means to have some individualistic touch". Kolarevic's (2015) perspective on design democratization can be framed as such menu picking activity, and instead of real democratization of the design process by transforming the user into a designer, the user becomes a consumer.

In a different perspective, Eric von Hippel (2005) credits the democratization of design to the radical and rapid improvement of the user's ability to innovate as a result of improvements on the quality of digital tools (hardware and software), access to easy-to-use tools and components for innovation, and access to a vibrant network of innovation commons. In architecture, for example, digital tools for design and prototyping were expensive assets frequently restricted to large offices. Nowadays, a 3D printer can be constructed at home using Lego and many CAD software based on explicit and parametric modeling are open source. Furthermore, there is a vast array of websites and design forums where designers share knowledge, designs, and codes. Within this context, Hippel $(2005$, p. 1) observes that "users that innovate can develop exactly what they want, rather than relying on manufacturers to act as their (often very imperfect) agents."

The idea of an "innovation user" relies on the fact that users have more information about their specific needs and use-context and as a result "tend to develop innovations that are functionally novel" (Hippel, 2005, p.8). The author, therefore, argues that companies and government fundings should shift from a manufacturercentric development system to a user-centered innovation process where "need-related innovation" tasks are outsourced to users. To enable this shift, users should be equipped with appropriate toolkits, defined as "integrated sets of product-design, prototyping, and design-testing tools intended for use by end users" (Hippel, 2005, p.8). Hippel (2005) question product configurators used by producers of mass customized products as it only offers a list of options that have been pre-designed by the mass customizer. Instead of choosing from a list, the idea of user-based customization via toolkits is to enable nonspecialist designers to design high-quality, producible custom products that exactly meet their needs. The concept of toolkit put forward by Hippel (2005) involves trial-and-error learning, user-friendly interfaces, libraries that can be incorporated into custom designs, the creation of solution space that encompasses the designs they want to create, and direct communication with the manufacturer's production equipment. This set of principles can be valuable for designers to design for design empowerment and democratization of the design process using parametric design and digital fabrication. However, some caution is needed, as the concept of innovation user and toolkit design were developed in order to offer a viable model for companies to adapt to a new context where users are already creating, sharing, and customizing their products and objects. If those processes already exist, it is important to understand how to potentialize it, instead of investing on another bias where large companies outsource the creative endeavor yet maintain the added value of the creation to centralize profit. 
In this context, the Wikihouse concept can offer valuable lessons. The Wikihouse process, as proposed by the English architects Alastair Parvin and Nick lerodiaconou, is characterized by the creation of design principles, the use of the SketchUp software, and the creation of a shared database (Parvin, 2013). The first design principles define which type of wood is to be used, how to think about the fitting of different elements and its resistance. These principles also cover how one should draw the elements in SketchUp. Parvin and Lerodiaconou developed a plug-in specially designed for SketchUp. This plug-in is presented as one of the significant advances of the system, as well as one of its advantages. In this manner, Wikihouse is not specifically a house, but an integrated set of processes that enables people to produce an architectural artifact.

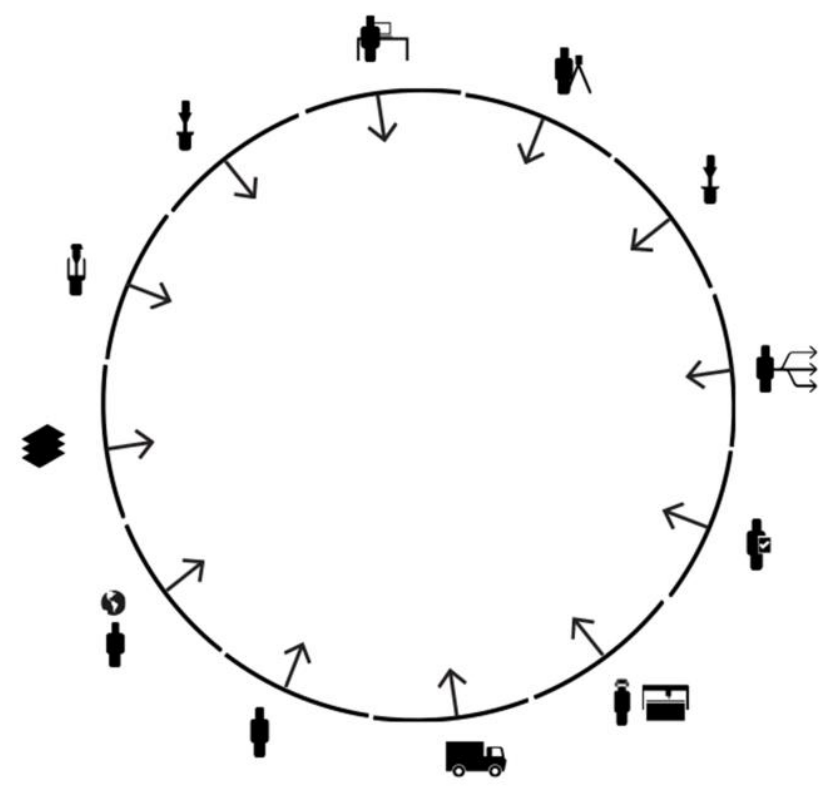

Figure 9: Image representing the concept of Wikihouse as a design community. (Wikihouse, n.d.).

If Wikihouse is compared to other systems such as Hermit House, many differences become clear. Wikihouse defines design principles while the Hermit House defines a shape. Although most of the principles of Wikihouse are essentially formal, there are concerns with user input in the design process, which opens possibilities for dialogue through the creation of communication channels. At the same time, it reveals the potential of parametric systems to trigger dialogues, especially if their underlying assumptions include the user as co-responsible for the production of space. Wikihouse is not a product configurator, nor it is a toolkit for housing design. It is not a finished idea to be applied nor a specific method for constructing a house. Wikihouse can be seen as a system with organizational closure (it is possible to identify it as a system) that involves design principles, design interfaces, companies, engineers, architects, lay users, researchers, and others, in a creative network. Understanding this systemic perspective may be key for designing towards true design democratization.

\section{DIGITAL CONVERSATIONS: FROM DIGITAL CONTINUUM TO CIRCULAR INFORMATION FLOWS}

According to Kolarevic (2003), one of the most profound aspects of contemporary architecture is the new found ability to generate construction information directly from design information through new processes and techniques of digital design and digital fabrication. For Kolarevic (2003, p.10), "when applied to architecture, the use of digital technologies raises not only the question of ideology, form or tectonics, but also the question of the significance of information, and, more importantly, who controls it." If parametric models become the primary source of information in design, analysis, fabrication, and construction, it would put the designer in a central position in the building process. The architect would perhaps even regain the absolute powers of the medieval master builder - becoming a "digital master builder" (Kolarevic, 2003). From that perspective, it seems that computational processes are interweaving bits and atoms in a continuous workflow of digital information.

The idea of a digital continuum may be attractive to many architects, as it gives them apparent control over the whole process. It suggests that the design process is flattened into a linear continuum, connecting the initial idea to product, which can be parametrically differentiated and digitally fabricated, which enables, among other things, mass customization. However, this perspective can be misleading and may diverge the focus from what can be a true paradigm shift in the design and fabrication process. Looking from the outside, the product of the digital process that combines parametric design and digital fabrication techniques can indeed suggest a continuous and linear process from design to production. Notwithstanding, an inside look reveals that both parametric design and digital fabrication are subjected to different contingencies inherent to the design process.

The design endeavor is a circular active conversation (with oneself or with others) where novelty is generated (Glanville, 2009), and that is not different with digital design processes. Glanville relates the distinction between a linear and circular perspective of the design with the image of a wheel leaving tracks in the sand. In this metaphor, someone looking from the inside will see that the wheel is the active design (research) process that is in a constant dynamic move, whereas someone looking from the outside may only see the linear trace left in sand - the product. Glanville (2000) points out that the problem is when the trace is taken for the wheel, becoming distortive and potentially prescriptive. Following this reasoning, from the outside the digital continuum may give the impression of a linear process, but an inside look reveals a process that involves circular conversations between the different craftsmen and stakeholders that are part of the design and building endeavor.

From that perspective, the notion of a linear and continuous digital process at an architectural scale gives a false idea of control that ignores the contingencies. In that sense, the digital continuum may not be much different from the perspectival paradigm that suggests a linear causal continuum between representation, making and use. Both notions disregards the social, political, and 
technical circumstances that frame architectural production. However, even if parametric design and digital fabrication does not eliminate contingencies, the notion of a digital workflow put forward by Scott Marble (2012) creates a different condition that favors the flow of digital information and the creation of networks within architecture, building, and society. Marble (2012) propose the shift of the focus from the potential of technological tools towards the importance of networks and information flows between design, assemble and industry.

\section{COPY-PASTE-SHARE}

Within the culture of parametric design, codes are being copied, edited, multiplied, and shared online in architectural forums and social media platforms. Because digital information can be copied without suffering losses and used for different purposes, designers explore digital collage to adapt the various algorithms to their goals and needs. This "copy-and-modify" strategy, as it is called by Woodbury (2010, p.38), is typically used because designers find it easier to change the code that works than to build it from scratch. Sometimes those copies gain their authenticity and enable a creative appropriation, but frequently they represent almost identical copies of the original code or process with small parameter changes. If it is said that frequently architects only build what they could draw, but in the new information environment that characterizes many parametric software, designers run the risk to only design what they can copy and modify. An example is a proliferation of "waffles" and "slices" in furniture and architectural design that frequently use the same original code. Leach (2016, p.219) points out that in digital culture "it is not the original that is important, but the number of times it is replicated. Originality has given way to replication and repetition".

However, there is a positive side to it. In a seminal article called Variety in Design, Glanville (1994, p.98) already acknowledged that "the making of perfect copies, the seamless collaging of elements from different sources, and the processes and actions of image transformation" were some of the particular strengths of digital processes. The ability to clone the original puts ownership in doubt, as any copy has the status of the original. What follows is that it is possible to originate things, but no longer own. The removal of the significance of ownership enables any part of anything to be shared, copied, cut, pasted, and transformed (Glanville, 1994). In a world of digital workflows, the sharing, copying, pasting, and editing of code can be seen as an opportunity for design conversations that embrace contingencies in the generation of novelty and can be seen as a possibility for democratizing design. If there is no more original and owner, everyone has virtually access to any design.

\section{FINAL CONSIDERATIONS}

The concepts of parametric design and digital fabrication were related to the notions of mass customization with its promise of design democratization. The difference between design configurators, meta-designs, meta-meta designs, and toolkits for user customization were discussed as a way to democratize design. It was possible to conclude that digital design interfaces for architecture increase the variety of solutions if they are based on principles that enable the intervention of those who inhabit the space, by giving them the means to act.
This ability can be seen as genuine democratization of the design process. However, to design those systems, a different approach is needed that understand all stakeholders, design interfaces, and fabrication processes as systems and subsystems that together enable the users to taka a creative part in the design process.

Although parametric design is not a new concept, its contemporary integration with digital fabrication is creating a new flux of digital information from design to use. It was observed that the notion of a linear and continuous digital process gives a false idea of control that eliminates contingencies. In a different path, the acceptance of the circular nature of design may lead to novel approaches where the contingent aspect of architecture is not seen as a problem, but as an opportunity for real design democratization. In that context, parametric design associated with digital fabrication in a mass-customization system can be used as a strategy to generate circular information flows that potentialize the different conversational cycles involved in designing and building, instead of trying to obscure them. Within this context design parameters should not only be redefined within parameters sliders but more importantly by the copying, transforming, and sharing of code.

\section{REFERENCES}

ASSA ASHUACH STUDIO @. (n.d.). Retrieved June 6, 2017, from http://assaashuach.com/

Aish. R, \& Woodbury, R. (2005). Multi-level Interaction in Parametric Design. In A. Butz. B. Fisher, A. Krüger, \& P. Olivier (Eds.), Smart Graphics (Vol. 3638. pp. 151-162). Berlin, Heidelberg: Springer Berlin Heidelberg. https://doi.org/10.1007/11536482_13.

Benjamin, D. (2012). Beyond Efficiency. In S. Marble (Ed.), Digital Workflows in Architecture (pp. 14-25). Basel: Birkhaeuser.

Davis, D. (2013). Modelled on Software Engineering: Flexible Parametric Models in the Practice of Architecture (Thesis). School of Architecture and Design: RMIT University, Melbourne.

Davis, S. (1997). Future Perfect: Tenth Anniversary Edition (Updated edition). Reading, Ma.: Basic Books.

Gerber, R. (2007) Parametric Practices: Models for Design Exploration in Architecture. Harvard University.

Glanville, R. (1994). Variety in design. Systems Research, 11(3), 95-103. https://doi.org/10.1002/sres.3850110307

Glanville, R. (1999). Researching Design and Designing Research. Design Issues, 15(2), 80. https://doi.org/10.2307/1511844

Glanville, R. (2000). The value of being unmanageable: Variety and creativity in cyberspace. Netzwerke, Falter Verlag, Vienna, 303-321.

Gramazio Kohler Architects ETH SIA BSA. (n.d.). Retrieved July 3, 2018, from http://gramaziokohler.com/web/e/projekte/17.html

Hippel, E. V. (2005). Democratizing Innovation (Administraıo edition). Cambridge, Mass.: The MIT Press.

Iwamoto, L. (2009). Digital fabrications: architectural and material techniques. New York: Princeton Architectural Press.

Kolarevic, B. (Ed.). (2003). Architecture in the digital age: design and manufacturing. New York, NY: Spon Press.
7 
Kolarevic, B. (2015). From Mass Customisation to Design "Democratisation." Architectural Design, 85(6), 48-53. https://doi.org/10.1002/ad.1976

Leach, N. (2016). The Culture of the Copy. Architectural Design, 86(5), 126-133. https://doi.org/10.1002/ad.2098

Marble, S. (Ed.). (2012). Digital Workflows in Architecture. Basel: Birkhaeuser.

Negroponte, N. (1975). Soft architecture machines. Cambridge, Mass: The MIT Press.

Nervous System. (n.d.). Retrieved May 6, 2017, from http://n-e-rv-o-u-s.com/

Noguchi, M., Formoso, C., da Rocha, C. G., Andújar-Montoya, M. D., Bunster, V., Cameron, R., \& Imai, C. (2016). Mass Customisation. In M. Noguchi (Ed.), ZEMCH: Toward the Delivery of Zero Energy Mass Custom Homes (pp. 95-119). Cham: Springer International Publishing. https://doi.org/10.1007/978-3-319-31967-4_4

Norman, D. A., \& Norman, D. (2005). Emotional Design: Why We Love (or Hate) Everyday Things. New York: Basic Books.
Pine, B. J. (1993). Mass Customization: The New Frontier in Business Competition (1 edition). Boston, Mass: Harvard Business Review Press.

Saul, G., Lau, M., Mitani, J., \& Igarashi, T. (2011). SketchChair: an all-in-one chair design system for end users (p. 73). ACM Press. https://doi.org/10.1145/1935701.1935717

Schumacher, P. (2008). Parametricism as Style: Parametricist Manifesto. Paper presented at The Darkside Club, 11th Architecture Biennale, Venice, 11 September 2008. Available at:. http://www. patrikschumacher.com/Texts/Parametricism as Style.htm. [Accessed 18 October 2017].

The Hermit Houses. (n.d.). Retrieved September 27, 2017, from http://www.hermithouses.nl/

WikiHouse. (n.d.). Retrieved July 3, 2018, from https://wikihouse.cc/

Woodbury, R. (2010). Elements of Parametric Design (1 edition). London; New York, NY: Routledge. 